\title{
Design of transport lines for uniforming beam distribution with octupoles
}

\author{
Y. Y. Guo ${ }^{1,2}$ and G. $\mathrm{Xu}^{1}$ \\ ${ }^{1}$ Institute of High Energy Physics, Chinese Academy of Sciences, Beijing 100049, China \\ ${ }^{2}$ University of Chinese Academy of Sciences, Beijing 100049, China
}

(Received 23 April 2012; published 7 February 2013)

\begin{abstract}
The intensity distribution of a typical particle beam is an approximate Gaussian distribution while many applications require the beam to be uniform. Local bright spots on the targets for high-power Gaussian beams create difficult cooling problems and shorten the lifetime of the target. In this paper, we have theoretically studied uniformization of the transverse beam profile using two octupole magnets placed at particular locations in the beam transport line. The details about the requirements on magnet strength and phase advance will be shown. As an example, we take a set of China Spallation Neutron Source parameters to simulate the beam. The simulation result agrees well with theoretical formulas.
\end{abstract}

DOI: 10.1103/PhysRevSTAB.16.023501

PACS numbers: 41.75. $-\mathrm{i}, 41.85 .-\mathrm{p}, 07.55 . \mathrm{Db}$

\section{INTRODUCTION}

Many charged-particle beam applications require illumination of the target with uniform beams. Local bright spots on the target create difficult cooling problems and shorten the lifetime of the target, so we need to adopt a certain approach to uniform the intensity distribution of the Gaussian beam to avoid the negative effect.

The idea that a system of controlled third-order aberrations produced by a pair of octupole magnets is able to substantially cause uniformization of the intensity distribution was first put forward by Philip F. Meads in 1983 [1]. Later, Sherrill and co-workers analytically verified that odd-order magnetic fields are needed to transform a Gaussian distribution into a uniform distribution [2]. In 1991, the Gaussian beam profile of the Brookhaven National Laboratory $200 \mathrm{MeV} \mathrm{H}^{-}$beam at the Radiation Effect Facility target was transformed into a rectangular profile with almost uniform distribution by placing two octupoles at particular locations along the beam transport line. It proved that a pair of octupoles can smooth the intensity distribution of a beam in the experiment [3]. This theory was also experimentally demonstrated in UNILAC [4]. The extracted beam from the Spallation Neutron Source at Oak Ridge National Laboratory is closed to a uniform beam in the horizontal direction [5]. In addition, Yuri has given the formulas that predict the strengths of the nonlinear magnetic fields required for beam uniformization [6]. In this paper, we have calculated the transverse coordinates of the beam at the target precisely using the transfer matrix method.

Published by the American Physical Society under the terms of the Creative Commons Attribution 3.0 License. Further distribution of this work must maintain attribution to the author(s) and the published article's title, journal citation, and DOI.
With the analysis of constraints, we can get the strength of octupole magnets and relevant parameters in the transport line in theory. Then we will give an application example.

\section{BEAM TRANSFORMATION}

We adopt the transport system as shown in Fig. 1. There is also a series of quadrupole magnets along the beam line that work together with octupole magnets in order to even the beam distribution.

We assume that the beam distribution is given by

$$
I\left(x_{0}\right)=I_{0} \exp \left(-\frac{x_{0}^{2}}{2 \sigma^{2}}\right) .
$$

Taking the thin lens approximation, the octupoles deflect the beam in proportion to the cube of the trajectory's displacement at the octupoles:

$$
\begin{array}{ll}
x=x_{0}, & x^{\prime}=x_{0}{ }^{\prime}-\frac{1}{6} K\left(x_{0}{ }^{3}-3 x_{0} y_{0}{ }^{2}\right), \\
y=y_{0}, & y^{\prime}=y_{0}{ }^{\prime}+\frac{1}{6} K\left(3 y_{0} x_{0}{ }^{2}-y_{0}{ }^{3}\right),
\end{array}
$$

where $K$ is the integral strength of the octupole.

$$
\text { 1st octupole 2nd octupole }
$$

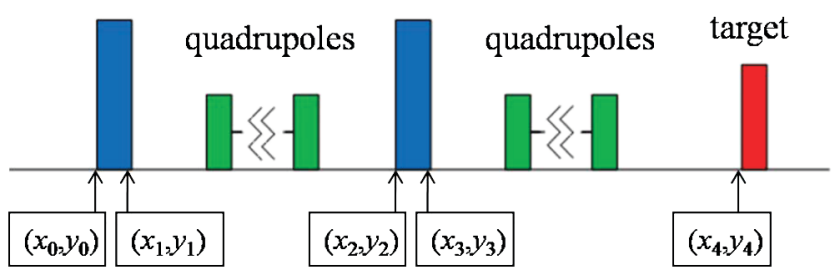

FIG. 1. A schematic plot of the transport system in the transport line. $x_{i}(i=0,1,2,3,4)$ stands for the displacement at different locations. 
According to the beam transport theory, we can get the beam displacement and angle at the target. In order to eliminate the higher than third-order terms, we can make the phase advance $n \pi$ ( $n \in$ integer) in both horizontal and vertical planes between the two octupoles [7]. In this condition, the transport matrix between the two octupoles is

$$
\left[\begin{array}{cccc}
a & 0 & 0 & 0 \\
b & \frac{1}{a} & 0 & 0 \\
0 & 0 & c & 0 \\
0 & 0 & d & \frac{1}{c}
\end{array}\right] .
$$

So we can get the displacement and angle at the exit of the second octupole:

$$
\begin{array}{ll}
x_{3}=a x_{0}, & x_{3}{ }^{\prime}=b x_{0}-\frac{K_{1}+a^{4} K_{2}}{6 a} x_{0}{ }^{3}+\frac{1}{a} x_{0}{ }^{\prime}+\frac{K_{1}+a^{2} c^{2} K_{2}}{2 a} x_{0} y_{0}{ }^{2}, \\
y_{3}=c y_{0}, & y_{3}{ }^{\prime}=d y_{0}-\frac{K_{1}+c^{4} K_{2}}{6 c} y_{0}{ }^{3}+\frac{1}{c} y_{0}{ }^{\prime}+\frac{K_{1}+a^{2} c^{2} K_{2}}{2 c} x_{0}{ }^{2} y_{0} .
\end{array}
$$

In Courant-Snyder parameters, $a, b, c$, and $d$ can be expressed as

$$
a= \pm \sqrt{\frac{\beta_{x 2}}{\beta_{x 1}}}, \quad b= \pm \frac{\alpha_{x 1}-\alpha_{x 2}}{\sqrt{\beta_{x 1} \beta_{x 2}}}, \quad c= \pm \sqrt{\frac{\beta_{y 2}}{\beta_{y 1}}}, \quad d= \pm \frac{\alpha_{y 1}-\alpha_{y 2}}{\sqrt{\beta_{y 1} \beta_{y 2}}},
$$

where \pm are for $n$ even or odd, respectively.

Here we make $\varphi_{x 1}=\pi, \varphi_{y 1}=\pi$. On the target, we have

$$
\begin{aligned}
& x_{4}=-x_{0}{ }^{\prime} \sqrt{\beta_{x 1} \beta_{x 3}} \sin \varphi_{x 2}-\frac{x_{0} \sqrt{\beta_{x 3}}\left(\cos \varphi_{x 2}+\alpha_{x 1} \sin \varphi_{x 2}\right)}{\sqrt{\beta_{x 1}}}+\frac{x_{0}{ }^{3}\left(K_{1} \beta_{x 1}{ }^{2}+K_{2} \beta_{x 2}{ }^{2}\right) \sqrt{\beta_{x 3}} \sin \varphi_{x 2}}{6 \beta_{x 1}{ }^{3 / 2}} \\
& -\frac{x_{0} y_{0}^{2} \sqrt{\beta_{x 3}}\left(K_{1} \beta_{x 1} \beta_{y 1}+K_{2} \beta_{x 2} \beta_{y 2}\right) \sin \varphi_{x 2}}{2 \sqrt{\beta_{x 1}} \beta_{y 1}}, \\
& x_{4}{ }^{\prime}=\frac{x_{0}{ }^{\prime} \sqrt{\beta_{x 1}}\left(-\cos \varphi_{x 2}+\alpha_{x 3} \sin \varphi_{x 2}\right)}{\sqrt{\beta_{x 3}}}-\frac{x_{0}\left[\left(\alpha_{x 1}-\alpha_{x 3}\right) \cos \varphi_{x 2}-\left(1+\alpha_{x 1} \alpha_{x 3}\right) \sin \varphi_{x 2}\right]}{\sqrt{\beta_{x 1} \beta_{x 3}}}+x_{0}{ }^{3}\left[\frac{K_{2} \beta_{x 2}{ }^{2}\left(\cos \varphi_{x 2}-\alpha_{x 3} \sin \varphi_{x 2}\right)}{6 \beta_{x 1}{ }^{3 / 2} \sqrt{\beta_{x 3}}}\right. \\
& \left.-\frac{K_{1} \sqrt{\beta_{x 1}}\left(-\cos \varphi_{x 2}+\alpha_{x 3} \sin \varphi_{x 2}\right)}{6 \sqrt{\beta_{x 3}}}\right]+x_{0} y_{0}{ }^{2}\left[-\frac{K_{1} \sqrt{\beta_{x 1}}\left(\cos \varphi_{x 2}-\alpha_{x 3} \sin \varphi_{x 2}\right)}{2 \sqrt{\beta_{x 3}}}+\frac{K_{2} \beta_{x 2} \beta_{y 2}\left(-\cos \varphi_{x 2}+\alpha_{x 3} \sin \varphi_{x 2}\right)}{2 \sqrt{\beta_{x 1}} \sqrt{\beta_{x 3}} \beta_{y 1}}\right] \\
& y_{4}=-\frac{y_{0} \sqrt{\beta_{y 3}}\left(\cos \varphi_{y 2}+\alpha_{y 1} \sin \varphi_{y 2}\right)}{\sqrt{\beta_{y 1}}}-y_{0}^{\prime} \sqrt{\beta_{y 1} \beta_{y 3}} \sin \varphi_{y 2}-\frac{x_{0}{ }^{2} y_{0} \sqrt{\beta_{y 3}}\left(K_{1} \beta_{x 1} \beta_{y 1}+K_{2} \beta_{x 2} \beta_{y 2}\right) \sin \varphi_{y 2}}{2 \sqrt{\beta_{y 1}} \beta_{x 1}} \\
& +\frac{y_{0}^{3}\left(K_{1} \beta_{y 1}^{2}+K_{2} \beta_{y 2}^{2}\right) \sqrt{\beta_{y 3}} \sin \varphi_{y 2}}{6 \beta_{y 1}^{3 / 2}}, \\
& y_{4}^{\prime}=\frac{y_{0}^{\prime} \sqrt{\beta_{y 1}}\left(-\cos \varphi_{y 2}+\alpha_{y 3} \sin \varphi_{y 2}\right)}{\sqrt{\beta_{y 3}}}-\frac{y_{0}\left[\left(\alpha_{y 1}-\alpha_{y 3}\right) \cos \varphi_{y 2}-\left(1+\alpha_{y 1} \alpha_{y 3}\right) \sin \varphi_{y 2}\right]}{\sqrt{\beta_{y 1} \beta_{y 3}}}+y_{0}^{3}\left[\frac{K_{2} \beta_{y 2}^{2}\left(\cos \varphi_{y 2}-\alpha_{y 3} \sin \varphi_{y 2}\right)}{6 \beta_{y 1}^{3 / 2} \sqrt{\beta_{y 3}}}\right. \\
& \left.-\frac{K_{1} \sqrt{\beta_{y 1}}\left(-\cos \varphi_{y 2}+\alpha_{y 3} \sin \varphi_{y 2}\right)}{6 \sqrt{\beta_{y 3}}}\right]+x_{0}{ }^{2} y_{0}\left[-\frac{K_{1} \sqrt{\beta_{y 1}}\left(\cos \varphi_{y 2}-\alpha_{y 3} \sin \varphi_{y 2}\right)}{2 \sqrt{\beta_{y 3}}}+\frac{K_{2} \beta_{x 2} \beta_{y 2}\left(-\cos \varphi_{y 2}+\alpha_{y 3} \sin \varphi_{y 2}\right)}{2 \sqrt{\beta_{y 1}} \sqrt{\beta_{y 3}} \beta_{x 1}}\right]
\end{aligned}
$$

where $K_{1}$ and $K_{2}$ are the integral strength of the first and the second octupole, $\beta_{x i}, \beta_{y i}, \alpha_{x i}, \alpha_{y i}(i=1,2,3)$ are the horizontal and vertical Courant-Snyder parameters, $i=$ 1, 2, 3 stand for location at the first octupole, the second octupole, and the target. $\varphi_{x 1}, \varphi_{y 1}$ are the horizontal and vertical phase advance between the two octupoles, $\varphi_{x 2}, \varphi_{y 2}$ are the horizontal and vertical phase advance between the second octupole and the target, $x_{0}, y_{0}, x_{0}{ }^{\prime}, y_{0}{ }^{\prime}$ stand for the displacement and angle just before the first octupole, and $x_{4}$, $y_{4}, x_{4}{ }^{\prime}, y_{4}{ }^{\prime}$ stand for the displacement and angle on the target.

When the phase advances are $n \pi$ ( $n \in$ integer) in both the horizontal and the vertical planes between the 
two octupoles, there are not any terms higher than 3 in the expression of the displacement and angle on the target. In the linear collider an octupole doublet can be used for tail folding, the phase advance between octupoles is close to zero, and the fifth-order terms are used to generate the aberration [8].

In order to eliminate the coupling term, we make $K_{1} \beta_{x 1} \beta_{y 1}+K_{2} \beta_{x 2} \beta_{y 2}=0$, or $K_{1}=-\frac{K_{2} \beta_{x 2} \beta_{y 2}}{\beta_{x 1} \beta_{y 1}}$, then we have

$$
\begin{aligned}
x_{4}= & -x_{0}{ }^{\prime} \sqrt{\beta_{x 1} \beta_{x 3}} \sin \varphi_{x 2}+\frac{K_{2} x_{0}{ }^{3} \beta_{x 2} \sqrt{\beta_{x 3}}\left(\beta_{x 2} \beta_{y 1}-\beta_{x 1} \beta_{y 2}\right) \sin \varphi_{x 2}}{6 \beta_{x 1}{ }^{3 / 2} \beta_{y 1}}-\frac{x_{0} \sqrt{\beta_{x 3}}\left(\cos \varphi_{x 2}+\alpha_{x 1} \sin \varphi_{x 2}\right)}{\sqrt{\beta_{x 1}}}, \\
x_{4}{ }^{\prime}= & \left.\frac{x_{0}{ }^{\prime} \sqrt{\beta_{x 1}}\left(-\cos \varphi_{x 2}+\alpha_{x 3} \sin \varphi_{x 2}\right)}{\sqrt{\beta_{x 3}}}-\frac{x_{0}\left[\left(\alpha_{x 1}-\alpha_{x 3}\right) \cos \varphi_{x 2}-\left(1+\alpha_{x 1} \alpha_{x 3}\right) \sin \varphi_{x 2}\right]}{\sqrt{\beta_{x 1} \beta_{x 3}}}\right] \\
& -x_{0}{ }^{3}\left[\frac{K_{2} \beta_{x 2}\left(\beta_{x 1} \beta_{y 2}-\beta_{x 2} \beta_{y 1}\right)\left(\cos \varphi_{x 2}-\alpha_{x 3} \sin \varphi_{x 2}\right)}{6 \beta_{x 1}{ }^{3 / 2} \sqrt{\beta_{x 3}} \beta_{y 1}}\right] \\
y_{4}= & -y_{0}{ }^{\prime} \sqrt{\beta_{y 1} \beta_{y 3}} \sin \varphi_{y 2}-\frac{K_{2} y_{0}{ }^{3} \beta_{y 2} \sqrt{\beta_{y 3}}\left(\beta_{x 2} \beta_{y 1}-\beta_{x 1} \beta_{y 2}\right) \sin \varphi_{y 2}}{6 \beta_{y 1}{ }^{3 / 2} \beta_{x 1}}-\frac{y_{0} \sqrt{\beta_{y 3}}\left(\cos \varphi_{y 2}+\alpha_{y 1} \sin \varphi_{y 2}\right)}{\sqrt{\beta_{y 1}}}, \\
y_{4}{ }^{\prime}= & \frac{y_{0}{ }^{\prime} \sqrt{\beta_{y 1}}\left(-\cos \varphi_{y 2}+\alpha_{y 3} \sin \varphi_{y 2}\right)}{\sqrt{\beta_{y 3}}}-\frac{y_{0}\left[\left(\alpha_{y 1}-\alpha_{y 3}\right) \cos \varphi_{y 2}-\left(1+\alpha_{y 1} \alpha_{y 3}\right) \sin \varphi_{y 2}\right]}{\sqrt{\beta_{y 1} \beta_{y 3}}} \\
& -\frac{K_{2} y_{0}{ }^{3} \beta_{y 2}\left(\beta_{x 1} \beta_{y 2}-\beta_{x 2} \beta_{y 1}\right)\left(-\cos \varphi_{y 2}+\alpha_{y 3} \sin \varphi_{y 2}\right)}{6 \beta_{y 1}{ }^{3 / 2} \beta_{x 1} \sqrt{\beta_{y 3}}} .
\end{aligned}
$$

\section{DISTRIBUTION OF CUBIC FUNCTION $g=x-k x^{3}$}

Assuming that the probability distribution of $x$ is Gaussian distribution of average 0 and variance $1: f(x)=\frac{1}{\sqrt{2 \pi}} e^{-\left(x^{2} / 2\right)}$. Now let us consider the probability distribution of $g$, where $g=x-k x^{3}$. The inverse function of $g=x-k x^{3}$ has three branches: $x 1=f 1(k, g), x 2=f 2(k, g), x 3=f 3(k, g)$. These branches are shown in Fig. 2: Figure 2 shows the sketch of inverse functions of $g=x-k x^{3}$. The branch points of the curve are $\pm \sqrt{\frac{4}{27 k}}$. So the probability distribution of $g$ is divided into three parts:

$$
\begin{aligned}
& f o 1(k, g)=-f(x 1) \frac{\partial x 1}{\partial g}, g<-\sqrt{\frac{4}{27 k}}, \\
& f o 2(k, g)=-f(x 1) \frac{\partial x 1}{\partial g}-f(x 3) \frac{\partial x 3}{\partial g}+f(x 2) \frac{\partial x 2}{\partial g},-\sqrt{\frac{4}{27 k}} \leq g \leq \sqrt{\frac{4}{27 k}}, \\
& f o 3(k, g)=-f(x 3) \frac{\partial x 3}{\partial g}, g>\sqrt{\frac{4}{27 k}} .
\end{aligned}
$$

We define $f o(k, g)=f o 2(k, 0),-\sqrt{\frac{4}{27 k}} \leq g \leq \sqrt{\frac{4}{27 k}}$.

When $k$ takes different values, the probability distribution of $g$ will be quite different. Figure 3 shows the probability distribution curve of $g$ when $k$ takes the value $0.14,0.095,0.032$, respectively. The black dashed curve is $f(x)$; the black curve is $f o(k, g)$ when $k=0.095$. The green curve is the probability distribution of $g$ when $k=$ $0.14, f o 1(0.14, g)$, and $f o 3(0.14, g)$ are very close to zero at this time; the middle part of $f o 2(0.14, g)$ is uniform, but it has a great value on both sides. The blue curve is the probability distribution of $g$ when $k=0.032$; the distribution is more similar to Gaussian distribution. The red curve is the probability distribution of $g$ when $k=0.095$. fo $1(0.095, g)$ and $f o 3(0.095, g)$ are also very close to zero at this time. In the range of $-\sqrt{\frac{4}{27 k}}$ to $\sqrt{\frac{4}{27 k}}$, the areas

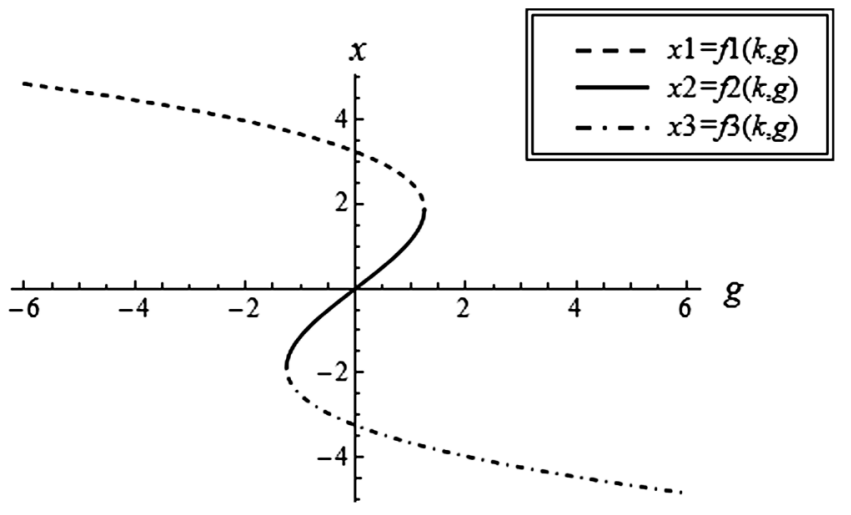

FIG. 2. The sketch of inverse functions of $g=x-k x^{3}$. The dashed curve is $f 1(k, g)$, the solid curve is $f 2(k, g)$, and the dotdashed one is $f 3(k, g)$. The branch points of the curve are $\pm \sqrt{\frac{4}{27 k}}$. 


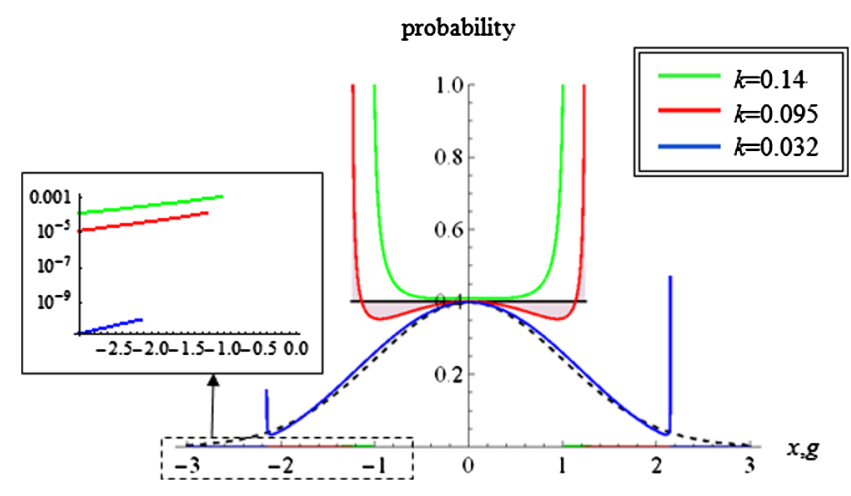

FIG. 3. The probability distribution of $g$ when $k=$ $0.14,0.095,0.032$, respectively. The black dashed curve is $f(x)$, the black curve is $f o(k, g)$ when $k=0.095$. The green curve is the probability distribution of $g$ when $k=0.14$, the blue curve is the probability distribution of $g$ when $k=0.032$ and the red one is the probability distribution of $g$ when $k=0.095$. The probabilities are very small when $g<-\sqrt{\frac{4}{27 k}}$ and $g>\sqrt{\frac{4}{27 k}}$. There logarithmic functions are shown in the box when $g<-\sqrt{\frac{4}{27 k}}$, the green curve, red curve, and the blue one are corresponding to $k=0.14,0.095,0.032$, respectively. The possibilities when $g>\sqrt{\frac{4}{27 k}}$ are symmetrical with that when $g<-\sqrt{\frac{4}{27 k}}$.

of shaded regions which are above and below the intersection of $f o 2(0.095, g)$ and $f o(0.095, g)$ are equal to each other. We think this is the most uniform distribution.

\section{APPLICATION EXAMPLE}

From the previous section, if the intensity distribution of $x_{0}$ is a Gaussian distribution of variance $\sigma$, then the distribution of $x=x_{0}-\frac{k}{\sigma^{2}} x_{0}{ }^{3}$ is most uniform when $k=0.095$. Take $\alpha_{x 1}=0, \alpha_{y 1}=0$, then the ratio of the third-order coefficient to the first-order coefficient in the expression of $x_{4}$ is

$$
\frac{K_{2} \beta_{x 2}\left(\beta_{x 1} \beta_{y 2}-\beta_{x 2} \beta_{y 1}\right) \sin \varphi_{x 2}}{6 \beta_{x 1} \beta_{y 1} \cos \varphi_{x 2}}=-\frac{0.095}{\beta_{x 1} \varepsilon} .
$$

The ratio of the third-order coefficient to the first-order coefficient in the expression of $y_{4}$ is similar:

$$
\frac{K_{2} \beta_{y 2}\left(\beta_{x 2} \beta_{y 1}-\beta_{x 1} \beta_{y 2}\right) \sin \varphi_{y 2}}{6 \beta_{x 1} \beta_{y 1} \cos \varphi_{y 2}}=-\frac{0.095}{\beta_{y 1} \varepsilon},
$$

where $\varepsilon$ is rms emittance.

Then we have

$$
\begin{aligned}
K_{2} & =-\frac{0.57 \beta_{y 1} \cos \varphi_{x 2}}{\beta_{x 2}\left(\beta_{x 1} \beta_{y 2}-\beta_{x 2} \beta_{y 1}\right) \sin \varphi_{x 2} \varepsilon} \\
& =-\frac{0.57 \beta_{x 1} \cos \varphi_{y 2}}{\beta_{y 2}\left(\beta_{x 2} \beta_{y 1}-\beta_{x 1} \beta_{y 2}\right) \sin \varphi_{y 2} \varepsilon} .
\end{aligned}
$$

So

$$
\frac{\beta_{y 1} \cot \varphi_{x 2}}{\beta_{x 2}}=-\frac{\beta_{x 1} \cot \varphi_{y 2}}{\beta_{y 2}}
$$

TABLE I. The CSNS RTBT parameters.

\begin{tabular}{lcc}
\hline \hline Parameter & \multicolumn{2}{c}{ Value } \\
\hline Beam energy $(\mathrm{GeV})$ & $E$ & 1.6 \\
Beam average current $(\mu \mathrm{A})$ & $I$ & 63 \\
Beam peak current $(\mathrm{A})$ & $I_{\max }$ & 3.6 \\
Acceptance $(\pi \mathrm{mm}$ mrad$)$ & $A$ & 350 \\
Emittance $(\pi \mathrm{mm}$ mrad, $\mathrm{rms})$ & $\varepsilon$ & 9 \\
Magnetic rigidity $(\mathrm{T} \mathrm{m})$ & $B \rho$ & 7.86707 \\
Target size $\left(\mathrm{mm}^{2}\right)$ & $a \times b$ & $120 \times 40$ \\
Thickness of VC and installation gap $(\mathrm{mm})^{\mathrm{a}}$ & $d$ & 5 \\
Pipe size $(\mathrm{mm})$ & $D$ & 250 \\
Field strength on pole of octupole $(\mathrm{T})$ & $B$ & 0.7 \\
Octupole length $(\mathrm{m})$ & $l$ & 0.6 \\
\hline \hline
\end{tabular}

${ }^{\mathrm{a}} \mathrm{V} \mathrm{C}$ stands for the vacuum chamber.

We can make $\beta_{x 1}=\beta_{y 2}, \beta_{y 1}=\beta_{x 2}, \cot \varphi_{x 2}=-\cot \varphi_{y 2}$ or $\varphi_{x 2}=\pi-\varphi, \varphi_{y 2}=\pi+\varphi$, and $\varphi$ is a very small value, then $K_{1}=-K_{2}$.

Here we take a set of China Spallation Neutron Source (CSNS) parameters to simulate the beam. The related CSNS ring to target beam transport (RTBT) parameters are shown in Table I.

The integral strength of the octupole must satisfy the following relation:

$$
\left|K_{1}\right|=\frac{6 B l}{B \rho\left(\sqrt{A \beta_{x 1}}+d\right)^{3}} .
$$

So

$$
\begin{aligned}
K_{2} & =-\frac{0.57 \beta_{y 1} \cot \varphi_{x 2}}{\beta_{x 2}\left(\beta_{x 1} \beta_{y 2}-\beta_{x 2} \beta_{y 1}\right) \varepsilon} \\
& =\frac{0.32}{\left(\sqrt{\beta_{x 1} \cdot 350 \times 10^{-6}}+0.005\right)^{3}} .
\end{aligned}
$$

Because $\beta_{x 2} \beta_{y 1} \ll \beta_{x 1} \beta_{y 2}$, we omit $\beta_{x 2} \beta_{y 1}$ in Eq. (13), so we can get a relational expression of $\varphi_{x 2}$ and $\beta_{x 1}$ :

$$
\varphi_{x 2}=\operatorname{Arccot}\left(-\frac{5057.72 \beta_{x 1}^{2}}{\left(\sqrt{350 \beta_{x 1}}+5\right)^{3}}\right) \text {. }
$$

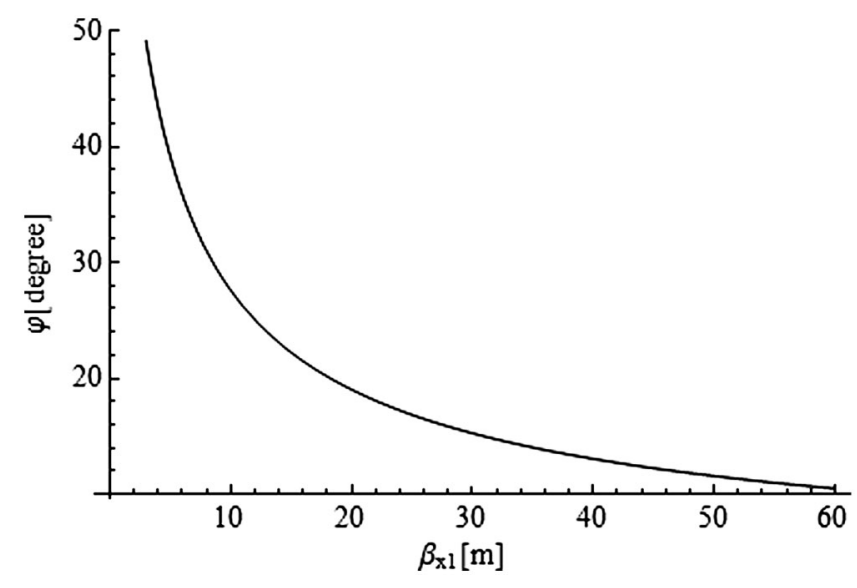

FIG. 4. The relational graph of $\varphi$ and $\beta_{x 1}$. 

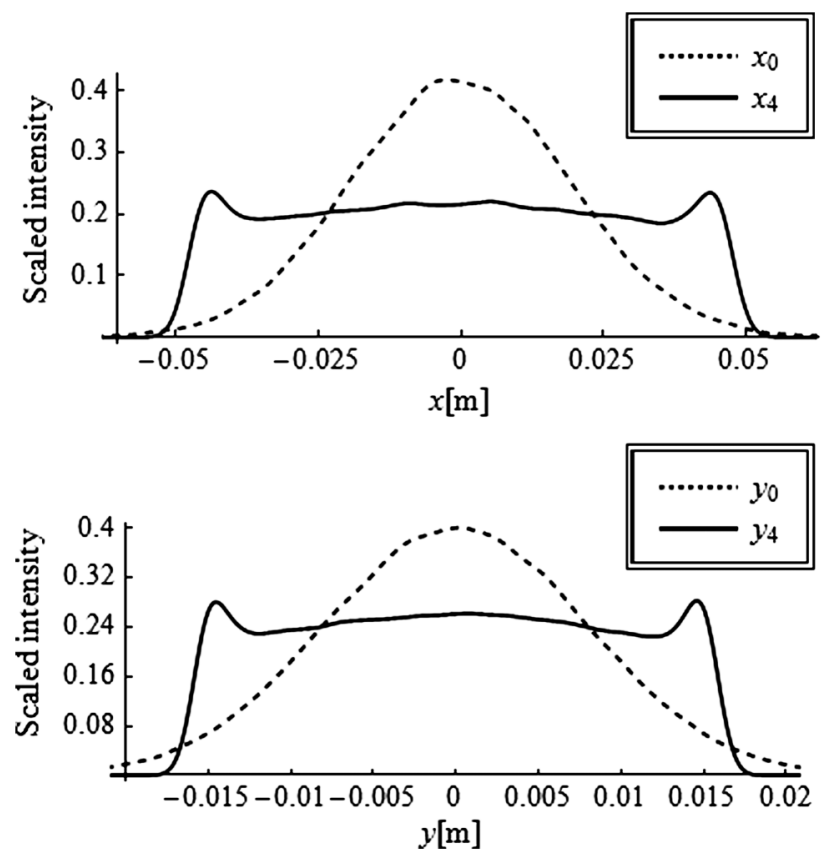

FIG. 5. Real-space scaled intensity distribution at the target in the horizontal direction (the upper plot) and the vertical direction (the bottom plot) when $\varphi=\frac{1}{180} \pi$. The dashed curve stands for the initial Gaussian distribution; the solid curve stands for the uniformed beam distribution.
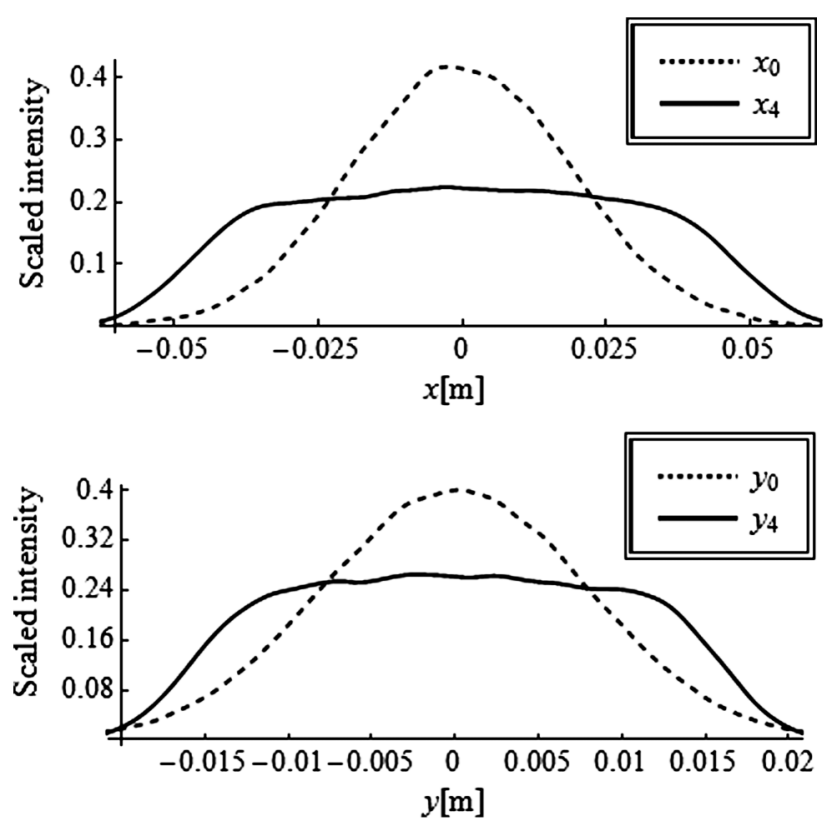

FIG. 6. Real-space scaled intensity distribution at the target in the horizontal direction (the upper plot) and the vertical direction (the bottom plot) when $\varphi=\frac{13.466}{180} \pi$. The dashed curve stands for the initial Gaussian distribution, the solid curve stands for the uniformed beam distribution, the slope of the solid curve is the impact of the $x_{0}{ }^{\prime}$ and $y_{0}{ }^{\prime}$ terms in the expression of $x_{4}$ and $y_{4}$.
That is,

$$
\varphi=\pi-\varphi_{x 2}=\pi-\operatorname{Arccot}\left(-\frac{5057.72 \beta_{x 1}^{2}}{\left(\sqrt{350 \beta_{x 1}}+5\right)^{3}}\right)
$$

The expression is shown in Fig. 4.

Because of the limit of the pipe size and the target size, here we take $\beta_{x 1}=\beta_{y 2}=40, \beta_{x 2}=\beta_{y 1}=7, \beta_{x 3}=$ $153, \beta_{y 3}=17$, then we can get $\varphi=\frac{13.466}{180} \pi$.

Now we will discuss the effect of $\varphi$ on the beam distribution individually. When we take $\varphi=\frac{1}{180} \pi$, the expressions of $x_{4}$ and $y_{4}$ are as follows:

$$
\begin{aligned}
& x_{4}=1.95546 x_{0}-516.025 x_{0}{ }^{3}-1.36531 x_{0}{ }^{\prime}, \\
& y_{4}=1.55815 y_{0}-2349.59 y_{0}{ }^{3}+0.190383 y_{0}{ }^{\prime} .
\end{aligned}
$$

The $x_{0}{ }^{\prime}$ and $y_{0}{ }^{\prime}$ terms are very small; the distribution of beam before and after uniforming is shown in Fig. 5.

Figure 5 shows the real-space scaled intensity distribution at the target in the horizontal direction and the vertical direction when $\varphi=\frac{1}{180} \pi$. The beam is uniform with al-

\begin{tabular}{|c|c|c|c|}
\hline Label & Element & Length (m) & Strength \\
\hline $\mathrm{O} 1$ & Octupole & 0 & -170.621 \\
\hline DOCT & Drift & 0.3 & \\
\hline D1 & Drift & 4.172 & \\
\hline Q1 & Quadrupole & 0.5 & 0.243 \\
\hline D2 & Drift & 4.084 & \\
\hline Q2 & Quadrupole & 0.5 & -0.332 \\
\hline D3 & Drift & 9.991 & \\
\hline Q3 & Quadrupole & 0.5 & 0.412 \\
\hline D4 & Drift & 0.759 & \\
\hline Q4 & Quadrupole & 0.5 & -0.412 \\
\hline D3 & Drift & 9.991 & \\
\hline Q5 & Quadrupole & 0.5 & 0.332 \\
\hline $\mathrm{D} 2$ & Drift & 4.084 & \\
\hline Q6 & Quadrupole & 0.5 & -0.243 \\
\hline D1 & Drift & 4.172 & \\
\hline DOCT & Drift & 0.3 & \\
\hline $\mathrm{O} 2$ & Octupole & 0 & 170.621 \\
\hline DOCT & Drift & 0.3 & \\
\hline D5 & Drift & 3.647 & \\
\hline Q7 & Quadrupole & 0.5 & -0.215 \\
\hline D6 & Drift & 2.455 & \\
\hline Q8 & Quadrupole & 0.5 & 0.641 \\
\hline D7 & Drift & 0.788 & \\
\hline Q9 & Quadrupole & 0.5 & -0.379 \\
\hline D8 & Drift & 4.649 & \\
\hline Q10 & Quadrupole & 0.5 & 0.513 \\
\hline D9 & Drift & 7.339 & \\
\hline Q11 & Quadrupole & 0.5 & -0.329 \\
\hline D10 & Drift & 12.462 & \\
\hline
\end{tabular}
most rectangular distribution after uniforming, but the

TABLE II. The lattice parameters. 

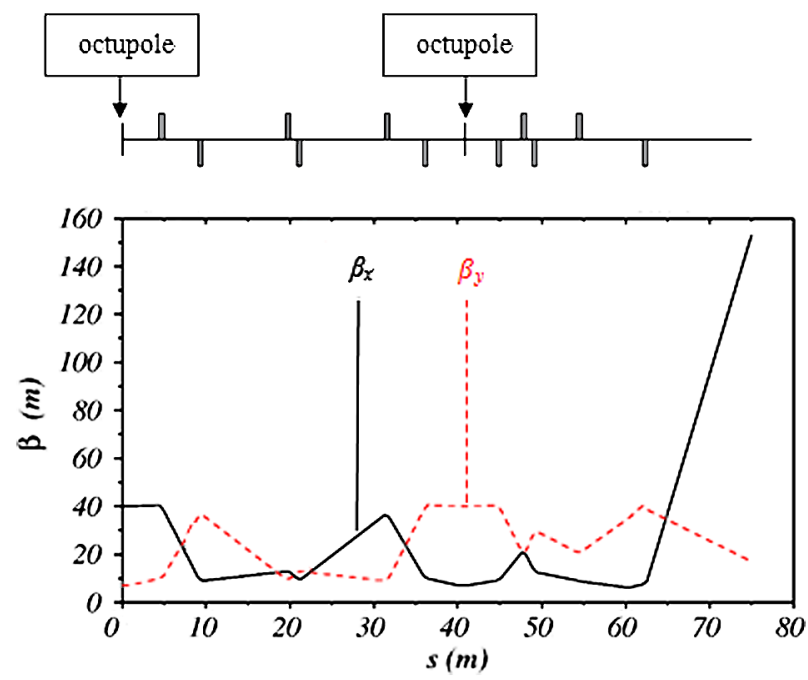

FIG. 7. Beam envelop.

particles do not completely cover the target and hit the target in the middle part of the region. In addition, the strength of the octupole is unavailable in this case. As we increase the value of $\varphi$ gradually, the area that the particles hit on the target becomes larger, and both sides of the rectangle start to become a slope. The reason is the impact of $x_{0}{ }^{\prime}$ and $y_{0}{ }^{\prime}$ terms.
When we take $\varphi=\frac{13.466}{180} \pi$, the expressions of $x_{4}$ and $y_{4}$ are as follows:

$x_{4}=1.90204 x_{0}-501.92929 x_{0}{ }^{3}-18.20830 x_{0}{ }^{\prime}$,

$y_{4}=1.51559 y_{0}-2285.41048 y_{0}^{3}+2.53902 y_{0}{ }^{\prime}$.

Figure 6 shows the initial distribution and the uniform distribution after optimizing in the horizontal direction and the vertical direction when $\varphi=\frac{13.466}{180} \pi$. The beam just can cover the target and the strength of the octupole is acceptable.

In this condition, we have done a particle-tracking simulation with MAD8 to compare the particle coordinates with what we get from the theory above. The lattice parameters are listed in Table II where we have taken the thin lens approximation and the strength of the octupole in Table II is the integral strength. The beam envelope graph is shown in Fig. 7.

In Table III, we give two sets of initial values $x_{0}, x_{0}{ }^{\prime}, y_{0}$, $y_{0}{ }^{\prime}$ arbitrarily and compare the particle coordinates through the four elements in Fig. 1 from the theoretical calculations above with the simulated results with MAD8 by thin and thick octupoles. From Table III, we can see that the particle coordinates from the theoretical calculations and simulation results in thin lens approximation are identical. On the other hand, although the results with thick octupoles are not exactly equal, they are very close.

TABLE III. The particle coordinates comparison of theory and simulation with MAD8.

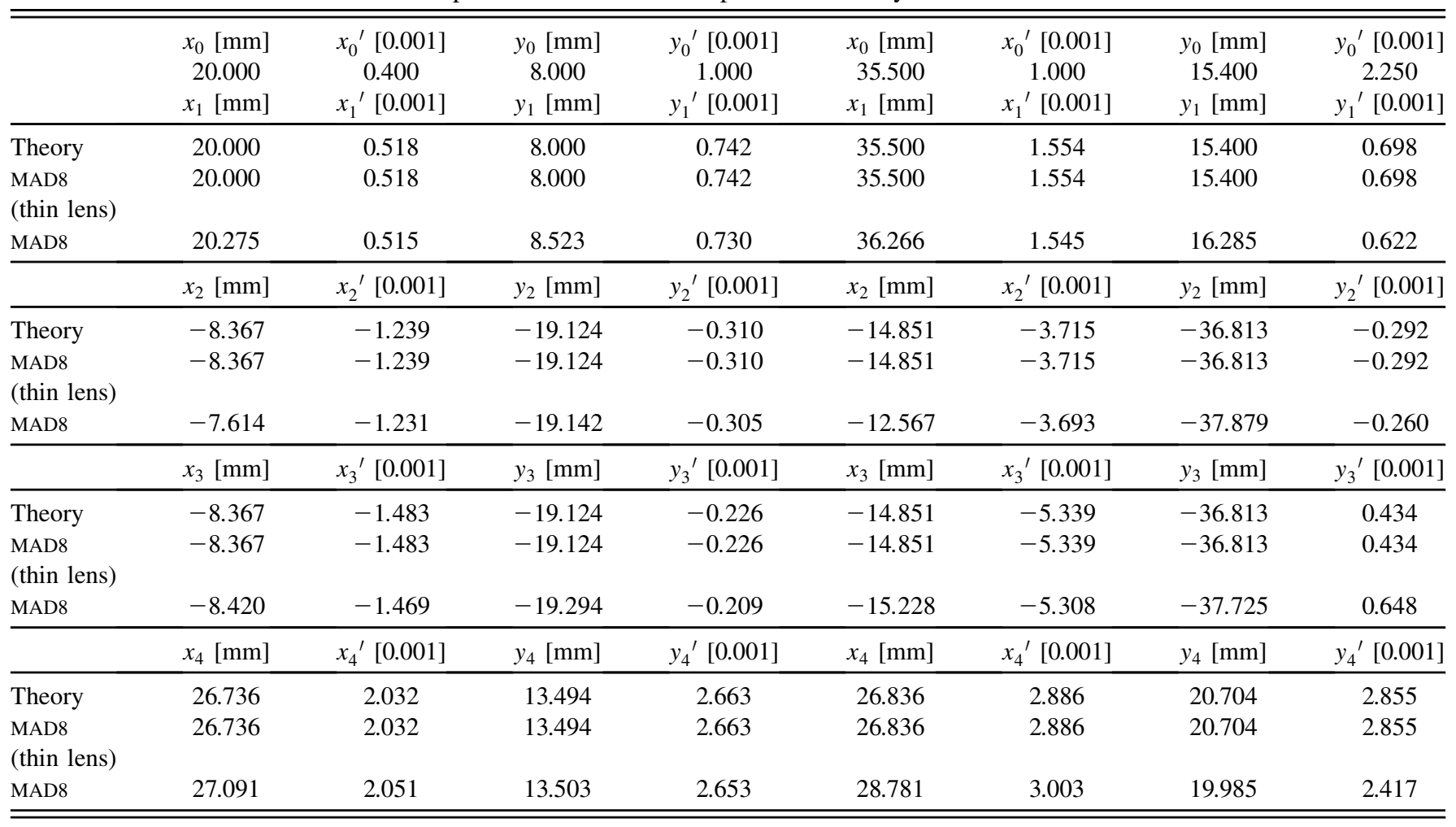




\section{SUMMARY}

In order to avoid the negative influence of the Gaussian beam to the target, we place an octupole system in the beam transport line. In the thin lens approximation, when the phase advances are $n \pi$ ( $n \in$ integer) in both horizontal and vertical planes between the two octupoles, the terms higher than 3 in the expressions of displacement and angle on the target will disappear, and if $K_{1}=-\frac{K_{2} \beta_{x 2} \beta_{y 2}}{\beta_{x 1} \beta_{y 1}}$, the coupling term will disappear, too. Moreover, the closer to $\pi$ the phase advances in the horizontal and the vertical plane between the second octupole and the target, the more uniform the distribution on the target, but the higher the strength of the octupole magnet.

We take the parameters of CSNS to simulate the beam with MAD8. The simulation results agree well with the theoretical formulas.

\section{ACKNOWLEDGMENTS}

The authors would like to thank Professor Wang, Professor Liu, Professor Huang, and N. Wang for their helpful suggestions and discussions.
[1] Philip F. Meads, IEEE Trans. Nucl. Sci. 30, 2838 (1983).

[2] B. Sherril, J. Bailey, E. Kashy, and C. Leakeas, Nucl. Instrum. Methods Phys. Res., Sect. B 40-41, 1004 (1989).

[3] N. Tsoupas, R. Lankshear, C. L. Snead, Jr., T. E. Ward, and M. Zucker, in Proceedings of the 1991 Particle Accelerator Conference, San Francisco, CA, 1991 (IEEE, New York, 1991).

[4] S. Richter and W. Barth, in Proceedings of the 8th European Particle Accelerator Conference, Paris, France, 2002 (EPS-IGA and CERN, Geneva, 2002), p. 1181.

[5] M Plum, in Proceedings of Hadron Beam 2008, Nashville, Tennessee [http://accelconf.web.cern.ch/AccelConf/HB2008/ papers/wgc04.pdf].

[6] Yosuke Yuri, Nobumasa Miyawaki, Tomihiro Kamiya, Watalu Yokota, Kazuo Arakawa and Mitsuhiro Fukuda, Phys. Rev. ST Accel. Beams 10, 104001 (2007).

[7] G. Xu, Phys. Rev. ST Accel. Beams 8, 104002 (2005).

[8] R. Brinkmann et al., in Proceedings of the Particle Accelerator Conference, Chicago, IL, 2001 (IEEE, New York, 2001). 